\title{
A histopathological study of cutaneous leishmaniasis in Sri Lanka
}

\author{
C H P Herath¹, N V I Ratnatunga², R Waduge ${ }^{3}$, P Ratnayake ${ }^{3}$, C N Ratnatunga ${ }^{4}$, S Ramadasa $^{2}$ \\ (Index words: cutaneous leishmaniasis, histopathology, leprosy, Sri Lanka)
}

\begin{abstract}
Introduction Cutaneous leishmaniasis is endemic in Sri Lanka. The immunopathogenesis of these lesions in Sri Lankans has not been documented.

Objectives To classify skin lesions into histological groups, to assess parasitic load, density of each inflammatory cell type and necrosis and to characterise the lymphocytic reaction in cutaneous leishmaniasis in comparison to leprosy.

Methods Skin biopsies from 31 patients with demonstrable amastigotes in smears or tissue sections were studied. The lesions were classified by two independent observers into four distinct histological groups based on different cell types in the inflammatory infiltrate and formation of granulomata. Parasitic load and the presence of necrosis were recorded. Immunohistochemical staining for CD45RO and CD20 for counting $T$ and $B$ cells respectively was done.
\end{abstract}

Results Histological groups of cutaneous leishmaniasis ranging from group I-IV were similar to that of the spectrum in leprosy ranging from lepromatous to tuberculoid leprosy. The histological groups from I-IV showed a significant inverse relationship with the mean parasitic index.

Necrosis was not a prominent feature. The mean percentage of $T$ cells in the histological spectrum from group I-IV in leishmaniasis was similar to the spectrum from lepromatous to tuberculoid leprosy. Mean percentage of T cells were $20.1 \%$ in group I, $20.5 \%$ in group II, $33.8 \%$ in group III and $47.8 \%$ in group IV. Lepromatous, borderline tuberculoid and tuberculoid leprosy had $21.3 \%$, $33.4 \%$ and $48.0 \% \mathrm{~T}$ cells respectively.

Conclusion Cutaneous leishmaniasis is a spectral disease similar to leprosy. The mean percentage $T$ cells from group I-IV were similar to those in the spectrum of leprosy and mean percentage B cells varied in a narrow range.

Ceylon Medical Journal 2010; 55: 106-11.

\section{Introduction}

Cutaneous leishmaniasis is endemic in Sri Lanka [1]. Pathogenesis of cutaneous leishmaniasis is a spectrum of immunological reaction varying from anergy in diffuse cutaneous leishmaniasis to hypersensitivity in the lupoid type. The histological picture of cutaneous leishmaniasis in some endemic areas can be classified into a spectrum as in leprosy [2]. Several histological classifications have been described consisting of 4-6 categories which varies from one endemic area to another [2-7]. It is timely to evaluate and document the histological appearances of cutaneous leishmaniasis in Sri Lanka. The wide histological spectrum of leishmaniasis could mimic other diseases like lupus vulgaris, acne and leprosy.

Many studies have indicated that activation of macrophages and a necrotizing process that destroys the macrophage with the parasite it contains, as being the mechanism involved in elimination of the parasite in cutaneous leishmaniasis [2, 8-11]. The objectives of this study were to document the histological features of cutaneous leishmaniasis and to classify cutaneous lesions into histological groups using criteria by Ridley [8], to assess the parasitic load, density of inflammatory cell types and necrosis in cutaneous lesions and to assess the immunophenotype of the lymphocytic infiltrate of cutaneous lesions in each histological group of cutaneous leishmaniasis in comparison to leprosy.

\section{Methods}

Thirty-one formalin fixed paraffin embedded, haematoxylin and eosin stained sections of skin biopsies with cutaneous leishmaniasis, proven by positive skin smears or demonstrable Leishmania amastigotes in histological sections were included.

Strept ABC envision method was used for immunohistochemistry. Cells for T cell lineage was detected with CD45RO (clone UCHL 1) and B cells with CD20 (clone L26). Seven cases of leprosy including the tuberculoid, borderline tuberculoid and lepromatous categories were also stained similarly. The staining for $\mathrm{T}$ cells in our cases of leishmaniasis and leprosy were compared with those of the $\mathrm{T}$ cell pattern stained for the CD3 marker [12].

Thirty-one lesions were classified according to modified classification originally proposed by Ridley [8].

Group I: Parasitized macrophages with variable lymphocytes and plasma cells.

${ }^{1}$ Department of Pathology, Teaching Hospital, Peradeniya, Sri Lanka, ${ }^{2}$ Department of Pathology, Faculty of Medicine, University of Peradeniya, Sri Lanka, ${ }^{3}$ Department of Pathology, General Hospital, Badulla, Sri Lanka and ${ }^{4}$ Teaching Hospital, Kandy, Sri Lanka.

Correspondence: CHPH, e-mail <chaturika_herath@yahoo.com>. Received 14 March and revised version accepted 3 July 2010. Competing interests: none declared. 
Group II: Parasitized macrophages with lymphocytes, plasma cells and ill formed histiocytic granulomata.

Group III: A mixture of macrophages (with or without parasites) lymphocytes, plasma cells and epithelioid granulomata.

Group IV: Epithelioid granulomatous response (with or without Langhans type multinucleated giant cells) with a few lymphocytes and plasma cells but no amastigotes.

Grouping was done by two independent observers and was statistically analysed using Kappa statistics to check the degree of inter-observer agreement. In the event of disagreement, the sections were reviewed simultaneously by both observers, and a consensus reached.

The cells in the inflammatory infiltrate were semiquantitatively assessed by calculating a mean cell score for each cell type as follows:

3 - Cell dominates the other cells

2 - The cell is in equal numbers to the other cells

$1-$ The cell is fewer in number than the other cell

0 - No cells

The cells thus assessed were macrophages, epithelioid cells, lymphocytes and plasma cells.

The parasitic load was evaluated per "standard" section according to modified Ridley's parasitic index. The mean parasitic index was calculated for each histological group.

6 - More than 100,000 amastigotes per standard section

5 - 10,001-100,000 amastigotes per standard section

4 - 1001-10,000 amastigotes per standard section

3 - 101-1000 amastigotes per standard section

2 - 11-100 amastigotes per standard section

1 - 1-10 amastigotes per standard section

0 - Amastigotes not seen

A "standard section" was a cut at $4 \mu$, completely occupied by a lesion of cutaneous leishmaniasis and occupying two fields under a $\times 10$ microscope objective. Since higher parasitic loads were uncountable, and as heavily parasitized macrophages contain about $10-20$ parasites and a $\times 40$ field completely occupied by such cells contained about 5000 parasites, a lesion uniformly composed of such cells represented 6 and a lesion with fewer cells represented 5. Parasitic index (PI) in relation to mean score of each cell type was analysed. Pearsons correlation coefficient for PI and each cell type was also statistically analysed.

Presence or absence of necrosis was documented as follows:

\section{Diffuse foci of necrosis \\ 1 Focal or individual cell necrosis \\ 0 No necrosis}

Epidermal changes and tissue reactions were also documented. The percentage of total T cells and B cells were obtained by counting five randomly chosen microscopic fields in the area of inflammatory infiltrate / granulomata in each case. Total cells and number of positive cells were counted in each field under oil immersion. Percentage of cells in a granuloma was obtained by dividing the number of positive cells by the total number of cells and multiplying by hundred.

\section{Results}

\section{Histological grouping}

Thirty one biopsies were classified by two independent observers and group I, II, III and IV had 10, 10,10 and 01 cases respectively. Inter-observer agreement on grouping was proven to be almost perfect (Kappa 0.85). Histology of lesions showed a spectrum ranging from diffuse parasitized macrophage infiltrate in group I to activated macrophages giving rise to well formed epithelioid tuberculoid granulomata in group IV. Histological groups of cutaneous leishmaniasis ranging from group I-IV were similar to that of the leprosy spectrum ranging from lepromatous to tuberculoid leprosy. Group IV lesions with granulomas resembled tuberculoid leprosy.

Table 1. Histological group, number of cases and parasitic index

\begin{tabular}{ccccc}
\hline Histological group & Number of cases & Parasitic index (mean) & SE of mean & Standard deviation \\
\hline I & 10 & 3.9 & 0.348 & 1.101 \\
II & 10 & 3.3 & 0.335 & 0.291 \\
III & 10 & 2.2 & & 0.919 \\
IV & 1 & 0.0 & & \\
\hline
\end{tabular}


Diffuse infiltrates of parasitized macrophages in group I to well formed epithelioid granulomata in group IV resembled that of diffuse infiltrate of foamy macrophages laden with bacterial globi in lepromatous leprosy to well formed tuberculoid granulomata in tuberculoid leprosy. The middle of the spectrum which comprised parasitized macrophages, lymphocytes, plasma cells with ill formed histiocytic granulomata in group II and parasitized macrophages admixed with epithelioid granulomata in group III were similar to borderline lepromatous and borderline tuberculoid leprosy.

API of $0,1,2,3,4,5$ and 6 was seen in 2, 0, 8, 12, 3, 6 and 0 cases respectively. There were no cases with PI of 1 and 6 . The majority of lesions showed a PI of 2 and 3. The mean PI along with the standard error of the mean and the standard deviation for each histological group is shown in Table 1. As shown in Figure 1, the histological groups from I-IV showed a significant inverse relationship with the mean PI (pearsons correlation coefficient $=-0.962, p=0.038$.)

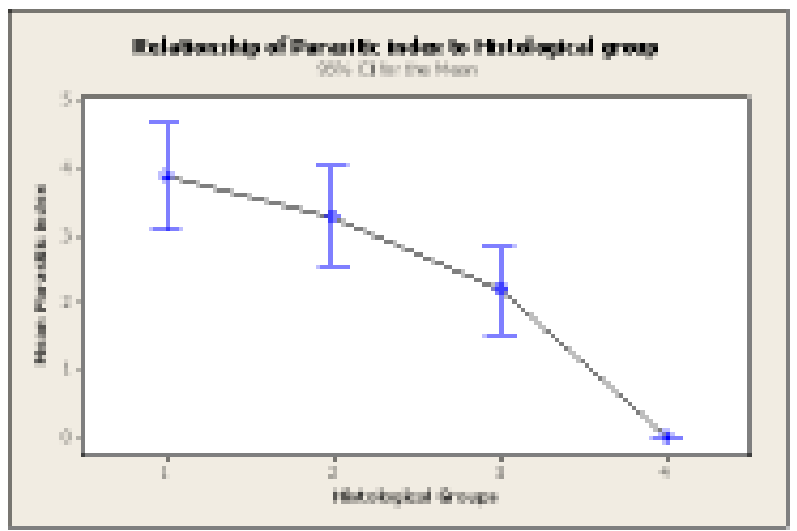

Figure 1. Relationship of parasitic index to histological group (pearsons correlation coefficient $=-0.962$, $p=0.038)$.

\section{Assessment of necrosis, epidermal changes and tissue reaction}

Necrosis was not a prominent feature. Eleven cases showed necrosis, 5 focal and 6 diffuse. Focal necrosis revealed foci of fibrinoid necrosis whereas diffuse necrosis revealed diffuse foci of nuclear debris with evidence of destruction of parasitized macrophages. The necrosis was seen in lesions with PI 2-5. Follicular plugging and epidermal atrophy were the major findings and were seen in 20 and 14 cases respectively. Parakeratosis, acanthosis and intra-epidermal abscesses were other findings.

One biopsy showed destruction of sebaceous glands. Six cases showed amastigotes within keratinocytes with possible trans-epidermal elimination of amastigotes. These epidermal changes are not seen in leprosy. Subepidermal upper dermal oedema was seen in 13 cases. Fibrosis was seen in 4 cases.

\section{Analysis of cellular infiltrate}

Semi-quantative analysis of the cellular infiltrate in relation to the PI showed a significant negative relationship with mean score for epithelioid cells and mean score for lymphocytes (both B and T cells) (Figure 2 and Table 2). The mean score for macrophages and plasma cells showed a significant positive relationship. (Figure 2 and Table 2)

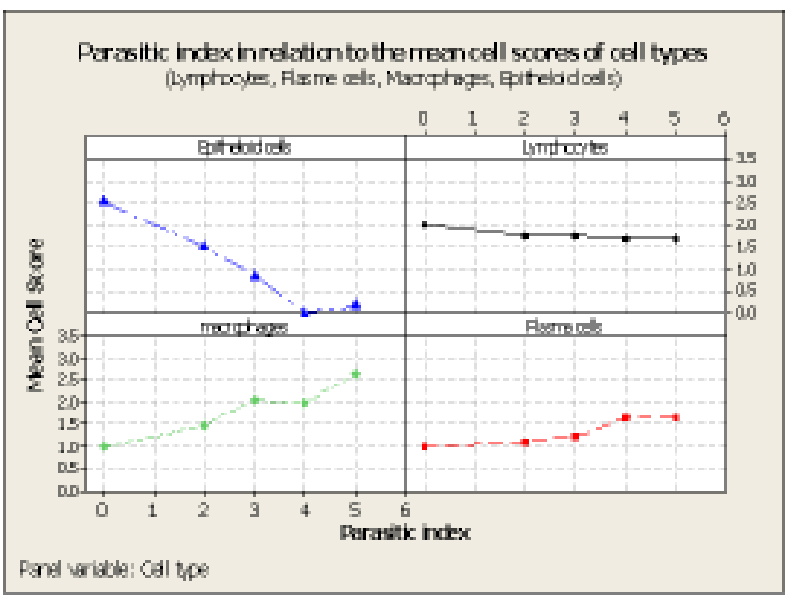

Figure 2. Mean cell score of epithelioid cells, lymphocytes, macrophages and plasma cells in lesions, in relation to parasitic index.

\section{Table 2. Pearsons correlation coefficient for parasitic index and each cell type}

\begin{tabular}{lcc}
\hline Cell types & Correlation coefficient & $p$ value \\
\hline Lymphocytes & -0.933 & 0.021 \\
Epitheliod cells & -0.970 & 0.006 \\
Plasma cells & 0.931 & 0.022 \\
Macrophages & 0.966 & 0.008
\end{tabular}

Immunohistochemistry revealed a predominant $\mathrm{T}$ cell mediated response from group I to IV as in leprosy. Mean percentage of T cells were $20.1 \%$ in group I, $20.5 \%$ in group II, $33.8 \%$ in group III and $47.8 \%$ in group IV. Mean percentage of B cells ranged from $10 \%$ to $4.6 \%$ from group I to IV. Lepromatous leprosy, borderline tuberculoid and tuberculoid leprosy had $21.3 \%, 33.4 \%$ and $48.0 \%$ T cells respectively and $\mathrm{B}$ cells ranged from $10.3 \%$ to $6.1 \%$. The mean percentage of $\mathrm{T}$ cells in leprosy lesions in this study were similar to that of mean percentage of T cells in leprosy spectrum assessed by Wesley et al using CD3 antibody [12].

The mean percentage of $\mathrm{T}$ cells in histological spectrum from group I to IV in leishmaniasis was similar to that of spectrum in leprosy from lepromatous to tuberculoid. 


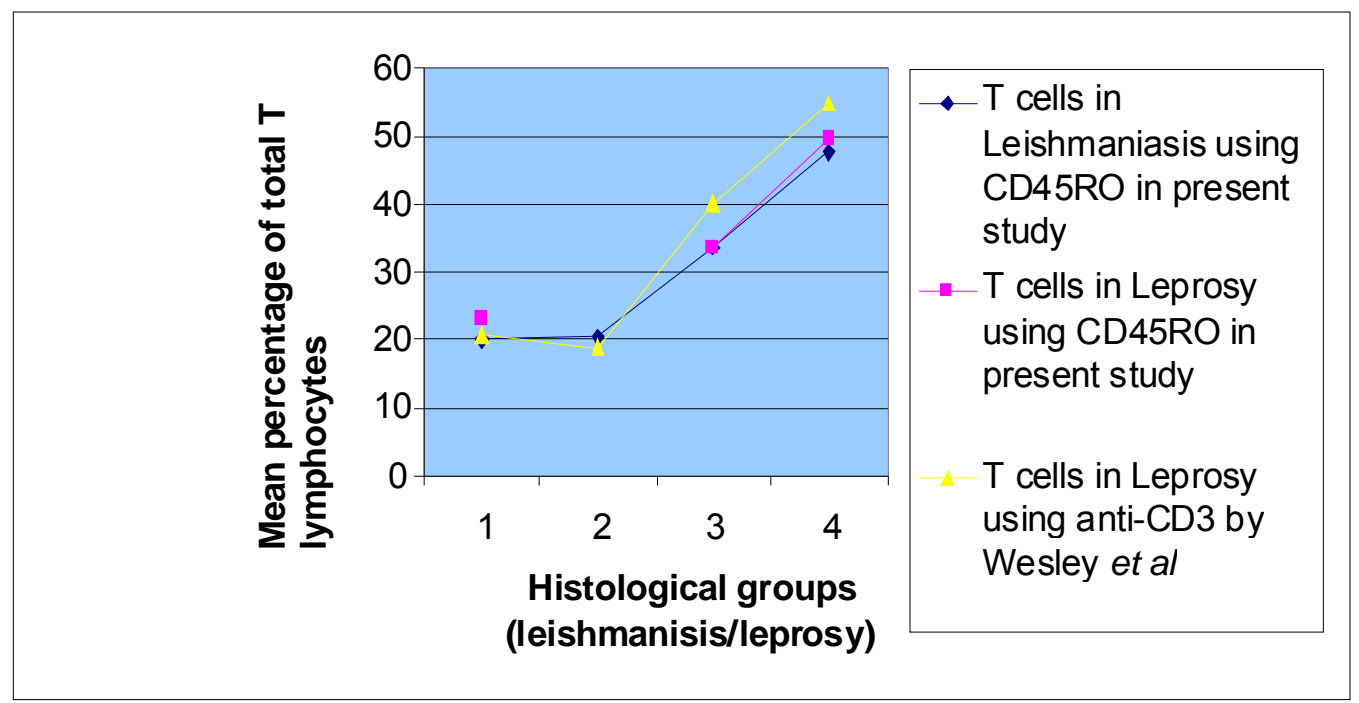

Figure 3. Comparison of mean percentage of total T cells in leishmaniasis and leprosy.

\section{Discussion}

Cutaneous leishmaniasis is endemic in Sri Lanka and this study was done to evaluate the histopathological features and host immune response in our population for better understanding of host parasite interaction and mechanisms of effective elimination of the parasite by the host. In many endemic areas detailed studies were performed with regard to histological features and were classified in to groups as it varies from region to region depending on the parasitic as well as host factors. To date no such study has been performed in Sri Lanka.

The four histological groups showed a linear spectrum in this study. Gaffar et al and Uthman et al studying cutaneous lesions from Sudan and Saudi Arabia respectively also classified lesions into four groups. In these studies, the groups I and IV were comparable to the polar forms of leprosy. The intermediate forms did not show a linear spectrum as in leprosy $[5,6]$. The spectrum and its variability are dependant on a number of factors such as the type and duration of clinical lesions, strain of organism, geographic location, parasitic load, and host immunity [7].

The spectrum of histological groups of cutaneous leishmaniasis in this study was similar to that of the leprosy spectrum. Diffuse infiltrates of parasitized macrophages in group I to well formed epithelioid granulomata in group IV resembled the diffuse infiltrate of foamy macrophages laden with bacterial globi in lepromatous leprosy to well formed tuberculoid granulomata in tuberculoid leprosy. The middle of the spectrum which comprised parasitized macrophages, lymphocytes, plasma cells with ill formed histiocytic granulomata in group II and parasitized macrophages admixed with epithelioid granulomata in group III were similar to borderline lepromatous and borderline tuberculoid leprosy. Ridley et al in 1978 studied cases of cutaneous leishmaniasis and leprosy seen at the Hospital for Tropical Diseases, London which represented all the main endemic areas of the world. Findings of Ridely et al with regard to histological groups were similar to the findings of Gaffar et al and Uthman et al, and concluded that the most important mechanism for elimination of parasites appeared to be necrosis of parasite laden macrophages [9].

Elimination of the parasite in cutaneous leishmaniasis involves two mechanisms, activation of intact macrophages and necrotizing process that destroys the macrophage with the parasite it contains. The latter is believed to be the more efficient mechanism for parasite elimination $[4,5]$. In this study necrosis was not a prominent feature as in other endemic areas and was only seen in eleven cases $(35.5 \%)$. This could be the reason for a linear spectrum in histological groups, the main mode of parasite elimination being macrophage activation giving rise to epithelioid granulomata.

The parasitic index from 0 - 6 showed a significant inverse relationship with the histological groups from group I to IV indicating a progressive reduction of parasites with evolution of the lesion from diffuse macrophage infiltrate to an epithelioid granulomatous response.

The mean score of epithelioid cells and lymphocytes showed a significant negative relationship with the parasitic index and macrophages showed a significant positive relationship. This also gives a clear indication of 
activated macrophages as the main mode of elimination of amastigotes in the lesions.

In a previous study necrosis was significantly associated with the presence of plasma cells [5]. The role of plasma cells in parasite elimination could not be established in this study. This may be due to insignificant necrosis in lesional biopsies. A few cases showed amastigotes within keratinocytes with a possible transepidermal elimination. Studies performed in inbred mice infected with Leishmania and lesional biopsies of humans have demonstrated trans-epidermal elimination of amastigotes [13,14].

One lesional biopsy revealed evidence of destruction of sebaceous glands. Involvement of dermal appendages and nerves by Mycobacterium leprae has been documented in leprosy, but not in cutaneous leishmaniasis [15, 16]. Superficial upper dermal oedema was a prominent tissue reaction evident in lesions. Fibrosis was only seen in four cases. Dermal oedema during the period of parasite destruction has been documented [10].

Leprosy is a spectral disease in which pathology and immunology are inextricably related, and it provides a unique critical model for investigating immunoregulatory mechanisms in humans [17]. The T cell mediated immune response plays an important role in leprosy [18]. There is a graded reduction in the number of T cells from tuberculoid to the lepromatous lesions [19]. In this study $\mathrm{T}$ cell mediated response with linear reduction from tuberculoid to lepromatous types was well demonstrated in leprosy lesions.

Granulomatous inflammatory infiltrate forms in response to a number of infectious agents [20]. The nature of cells comprising this infiltrate has been an area of active investigation in humans and murine models and the T cell mediated immune response plays a central role in the outcome of the disease [21-24]. Activation of macrophages is immunologically mediated and involves T cells [5]. Further studies have shown $\mathrm{T}$ cell mediated IFN- $\gamma$ and TNF- $\alpha$ which activate parasite infected macrophages for parasite destruction [25]. The mean percentage of T cells was $20 \%, 20.5 \%, 33.8 \%$ and $47.8 \%$ in groups I, II, III and IV respectively. Mean percentage of T cells increased in groups I to IV and were comparable to the mean percentage of T cells from lepromatous to tuberculoid leprosy lesions.

In conclusion, histological groups of cutaneous leishmaniasis in our population are similar to that of leprosy suggestive of a similar immunopathological process.

\section{References}

1. Samaranayake TN, Fernando SD, Jayasekara RW, Dissanayake VHW. Host genetic susceptibility to cutaneous leishmaniasis in Sri Lanka. Ceylon Medical Journal 2010; 55 (Supp): 29.
2. Azadeh B, Samad A, Ardehali S. Histological spectrum of cutaneous leishmaniasis due to Leishmania tropica. Transactions of Royal Society of Tropical Medicine and Hygiene 1985; 79: 631-6.

3. Bryceson ADM. Diffuse cutaneous leishmaniasis in Ethiopia. Transactions of Royal Society of Tropical Medicine and Hygiene 1969; 63: 708-36.

4. Ridley DS, Marsden PD, Cuba CC, Barreto AC. A histopathological classification of mucocutaneous leishmaniasis in Brazil. Transactions of Royal Society of Tropical Medicine and Hygiene 1980; 74: 508-14.

5. Gaafar A, Kadaro EL, Theander TG, et al. The pathology of cutaneous leishmaniasis due to Leishmania major in Sudan. American Journal of Tropical Medicine and Hygiene 1995; 52: 438-42.

6. Uthman MAE, Satir AA, Tabara KS. Clinical and histopathological features of zoonotic cutaneous leishmaniasis in Saudi Arabia. Journal of the European Academy of Dermatology and Venereology 2005; 19: 431-6.

7. Mysore V, Mohommed M, Leena D. Histopathological spectrum in cutaneous leishmaniasis: a study in Oman. Indian Journal of Dermatology, Venereology and Leprology 2001; 67: 294-8.

8. Ridley DS, Peters W, Killick-Kendrick R eds. The Leishmaniases in Biology and Medicine. V.2. London: Academic Press. 1987; 665-702.

9. Ridley DS. A histological classification of cutaneous leishmaniasis and its geographical expression. Transactions of Royal Society of Tropical Medicine and Hygiene 1980; 74: 515-21.

10. Ridley DS, Ridley MJ. The evolution of the lesion in cutaneous leishmaniasis. Journal of Pathology 1983; 141: 83-96.

11. Ridley DS. The pathogenesis of cutaneous leishmaniasis. Transactions of Royal Society of Tropical Medicine and Hygiene 1979; 73: 150-60.

12. Wesley $\mathrm{C}$ et al. The cutaneous infiltrate of leprosy. New England Journal of Medicine 1982; 307: 1593-7.

13. Vasconcellos C, Kauffman MR, Stto MN. Persistence of Leishmania antigen in C57BI/6j inbred mice infected with Leishmania amazonensis. Journal of the Brazilian Medical Association 1999; 45: 225- 8.

14. Puig L, Pradinaud R. Leishmania and HIV co-infection: dermatological manifestations. Annals of Tropical Medicine and Parasitology 2003; 97: 107-14.

15. Lever W, Lever GS. Histopathology of the skin. 7th edn. London, New York, Sydney. J. B. Lippincott Company 2005; 569-77.

16. Weedon W. Skin Pathology. London, New York, Tokyo. Churchill Livingstone 1997; 533-6.

17. Modlin RL. Learning from lesions: patterns of tissue inflammation in leprosy. Proceedings of the National Academy of Sciences of the United States of America 1988; 85: 1213-7.

18. Mahaisavariya P, Kulthanan K, Khemngern MA, Pinkaew $\mathrm{S}$. Lesional $\mathrm{T}$ cell subset in leprosy and leprosy reaction. International Journal of Dermatology 1999: 38: 345-8. 
19. Nath I. Immunology of leprosy - current status. Leprosy Review 1983; 31-45.

20. Rahman SB, Bari A. Composition of T cell immune response in cutaneous leishmaniasis. Journal of Pakistan Association of Dermatologists 2005; 15: 296-303.

21. Da-Cruz AM, Conceilcao-Silva F, Bertho AL, Coutinho SG. Leishmania reactive CD4+ and CD8 $+\mathrm{T}$ cells associated with cure of human cutaneous leishmaniasis. Infection and Immunity 1994; 62: 2614-8.

22. Rogers KA, Titus RG. Characterisation of the early cellular response to Leishmania major using peripheral blood mononuclear cells from Leishmania naïve humans. American Journal of Tropical Medicine and Hygiene 2004: 71: 568-76.

23. Isaza DM, Restrepo M, Restrepo R, Caceres-Dittmar G,
Tapia F. Immunocytochemical and histopathological characterisation of lesions from patients with localised cutaneous leishmaniasis caused by Leishmania panamensis. American Journal of Tropical Medicine and Hygiene 1996; 55: $365-9$.

24. Lima HC, Vasconcelos AW, David JR, Lerner EA. American cutaneous leishmaniasis of the cellular immune response with time. American Journal of Tropical Medicine and Hygiene 1994; 50: 743-7.

25. Antonelli LRV, Dutra WO, Almeida RP, Bacellar O, Gollob $\mathrm{KJ}$. Antigen specific correlation of cellular responses in human leishmaniasis suggests mechanisms for immunoregulation. Clinical and Experimental Immunology 2004; 136: $341-9$. 